\title{
Specific Volume and Degree of Crystallinity of Semicrys- talline Poly(chlorotrifluoroethylene), and Estimated Specific Volumes of the Pure Amorphous and Crys- talline Phases
}

\author{
John D. Hoffman and James J. Weeks
}

\begin{abstract}
The specific volume of poly (chlorotrifluoroethylene) has been measured from $-40^{\circ}$ to $+260^{\circ} \mathrm{C}$. Both quenched and well-crystallized specimens prepared by reproducible procedures were studied. Well-defined glass transitions were found close to $52^{\circ} \mathrm{C}$ in both specimens. Hence the glass temperature of this polymer is essentially independent of the degree of crystallinity. The quasi-equilibrium melting point of the particular type of crystallized specimen used in the investigation was $216^{\circ} \mathrm{C}$. The equilibrium melting temperature is undoubtedly somewhat higher, and probably lies between $220^{\circ}$ and $225^{\circ} \mathrm{C}$.

The degree of crystallinity of the crystallized and quenched specimens was calculated at $T_{\mathrm{g}}$ from specific-volume measurements alone, using a straightforward thermodynamic procedure. The method avoids a long "unguided" extrapolation of the liquid data to low temperatures, and does not involve the inexact approximation that the volume-temperature derivative of the glassy and crystalline states are the same. It has the further advantage of not requiring a pure crystal density from another source, such as a unit-cell determination from X-ray data. It was found that the quenched sample was 39 percent crystalline and the well-crystallized one 82 percent. A simple extension of the theory permits the degree of crystallinity to be computed as a function of temperature. The results are compared with those obtained in earlier investigations.

The specific volumes and volume-temperature derivatives of the pure supercooled liquid, glassy, and crystalline phases are estimated over a wide range of temperature. Certain quantities related to the free volume of the glassy state are discussed.

The methods outlined may be of utility in analyzing specific-volume-temperature data on other semicrystalline polymers where the rapid onset of crystallization interferes with a direct study of the supercooled liquid and glassy states, and where independent data on the properties of the pure crystalline phase are not available.
\end{abstract}

\section{Introduction}

Poly (chlorotrifluoroethylene) is a comparatively high-melting and semicrystalline polymer whose degree of crystallinity is strongly dependent on thermal history, samples rapidly quenched from the melt to room temperature being considerably less crystalline than those cooled slowly from the melt. Knowledge of the degree of crystallinity of this material is of interest in the analysis of its physical properties because these frequently depend on the extent of crystallization. For example, the mechanical [1] ${ }^{1}$ and dielectric [2] properties of highly crystallized and strongly quenched specimens are decidedly different. One of the principal objectives of the present research was to determine the degree of crystallinity of both strongly quenched and highly crystallized specimens prepared by procedures that could easily be reproduced in any laboratory. A refined method of analysis based on specific-volumetemperature measurements is used to obtain the degree of crystallinity of the samples. Information obtained from this study has been used in the analysis of dielectric [2] and rate-of-crystallization [3] data on specimens of poly(chlorotrifluoroethylene) from the same source as that employed in the present research.

Previous estimates of the degree of crystallinity of poly(chlorotrifluoroethylene) as a function of temperature for both quenched and slow-cooled

${ }^{1}$ Figures in brackets indicate the literature references at the end of this paper. samples have been obtained by Hoffman [4], using specific-heat data, and by Matsuo $[5,6]$, using an infrared method. Also, Price [7] has determined the degree of crystallinity of a crystallized specimen of this polymer as a function temperature, using a simple but approximate method based on specificvolume-temperature measurements.

Although the results of the various investigators mentioned above are in fair agreement, there are good reasons for redetermining the degree of crystallinity of this polymer by the proposed method. First, the particular crystallization and quenching procedures used in the aforementioned investigations might well have led to sample variations that would account for at least part of the differences in the results, and it would undoubtedly be better to compare the results of various methods by using specimens prepared in a more definite and reproducible manner. Also, it is clear that it would be advantageous to deal with a more highly crystalline specimen than was formerly used in order to determine the properties of the completely crystalline material more precisely. Further, the proposed method of determining the degree of crystallinity is capable of yielding more accurate results than those obtained by previous methods based on specific-volume measurements because certain approximations inherent in these methods are removed. Another point is that most of the determinations of the degree of crystallinity mentioned above were made without knowledge of the glass-transition temperature of this polymer. The situation concerning the glass 
transition in this polymer has been recently clarified by Mandelkern, Martin, and Quinn [8], who conclusively demonstrated the existence of such an effect about $20^{\circ} \mathrm{C}$ above room temperature. Lack of knowledge of the general whereabouts of the glass transition will lead to a rather large uncertainty in a determination of the degree of crystallinity based on specific-volume measurements [7]. Finally, it was considered to be of interest to determine whether or not specific-volume measurements at room temperature could be used to measure the degree of crystallinity of this material for the reason that such a method would be both simple and of general utility. It was found that this type of determination could be successfully carried out.

As implied above, it is necessary to know the glasstransition temperature, $T_{g}$, for a polymer in order to determine the degree of crystallinity by a method based on specific volume measurements. In the course of this investigation $T_{g}$ was measured by a method based on a comparison of the specific-volume-temperature curves for highly crystallized and strongly quenched specimens. The quasi-equilibrium melting temperature of the crystallized sample was also measured and compared with values obtained in other investigations.

Entirely apart from the degree of crystallinity, other information concerning poly(chlorotrifluoroethylene) is obtained from the measurements. A direct experimental study of most of the pure supercooled liquid range is prevented by the rapid onset of crystallization as the material is cooled from the melt; furthermore, samples of ordinary thickness cannot be quenched rapidly enough to obtain the pure crystal-free glass. The purely crystalline state is also not obtained in isolated form owing at least partly to the extraordinary slowness of the crystallization process when the degree of crystallinity becomes fairly high. However, by a study of the present type on two samples of widely differing degrees of crystallinity, it is possible to obtain quantitative information concerning the specific volumes and volume-temperature derivatives of the pure supercooled liquid, glassy, and crystallines states. A fairly precise estimate of the decrease of fractional free volume in the pure glassy state as the temperature is lowered is obtained.

\section{Experimental Procedure}

\subsection{Materials}

The poly(chlorotrifluoroethylene) used in this investigation was Kel-F grade 300 polymer, which was kindly supplied in sheet form by H. S. Kaufman of the Minnesota Mining \& Manufacturing Co. The sheets were approximately 1.5 to $3.0 \mathrm{~mm}$ thick. The number average molecular weight of the polymer was stated to be approximately 415,000 .

\subsection{Preparation of Crystalline Samples}

A simple crystallization procedure of reasonably short duration that yields reproducible results is to first melt out all the crystals by heating to $250^{\circ} \mathrm{C}$, and then to bring the specimen successively to temperatures of $200^{\circ}$ and $190^{\circ} \mathrm{C}$ for 1 day each, and finally to $180^{\circ} \mathrm{C}$ for 3 days. The material is then allowed to cool to room temperature over a period of a few hours. The long residence time at high temperatures permits the slow second stage [3] of the crystallization to proceed to an appreciable degree. The samples were crystallized in an atmosphere of nitrogen. Polymer crystallized by this technique is referred to in the text as "5-day" material. Specimens crystallized in this particular manner have a cloudy-white appearance. ${ }^{2}$ The specific volume is $0.4620 \mathrm{~cm}^{3} \mathrm{~g}^{-1}$ at $25^{\mathrm{C}} \mathrm{C}$. Samples from different batches of polymer crystallized by using the 5-day procedure rarely exhibited specific volumes that deviated more than $0.0003 \mathrm{~cm}^{3} \mathrm{~g}^{-1}$ from this value at $25^{\circ} \mathrm{C}$

A strictly isothermal run of even several weeks duration at either $180^{\circ}, 190^{\circ}$, or $200^{\circ} \mathrm{C}$ is less effective in producing highly crystalline material than the above-mentioned procedure lasting 5 days, which involves three different temperatures.

\subsection{Preparation of Quenched Samples}

Quenched samples were prepared by heating portions of sheet polymer $1.5 \mathrm{~mm}$ thick, which were clamped between 1-mm gold-plated copper disks, to $250^{\circ} \mathrm{C}$, and then dropping the entire assembly into ice water. It was found that the specific volume of such specimens was $0.4727 \mathrm{~cm}^{3} \mathrm{~g}^{-1}$ at $25^{\circ} \mathrm{C}$, the variation between samples generally being less than 0.0004 $\mathrm{cm}^{3} \mathrm{~g}^{-1}$. Mandelkern and co-workers found a value of $0.4739 \mathrm{~cm}^{3} \mathrm{~g}^{-1}$ at $25^{\circ} \mathrm{C}$ for a $3-\mathrm{mm}$ sheet of polymer quenched by an efficient procedure $\mid 8] .^{3}$ The specific volume of the $3-\mathrm{mm}$ sheet as supplied, which was simply removed from the hot mold and quenched in air, was generally only about 0.0005 to $.0010 \mathrm{~cm}^{3} \mathrm{~g}^{-1}$ lower than that produced by quenching in ice water. Quenched samples are optically clear.

\subsection{Specific-Volume Measurements}

The specific volume of the polymer was measured at various temperatures between $-40^{\circ}$ and $+260^{\circ} \mathrm{C}$, using a buoyancy method similar in many respects to that employed by Price [1]. Each polymer specimen used weighed approximately $5 \mathrm{~g}$ in air. The samples were suspended from a $0.1-\mathrm{mm}$ diameter Chromel A wire and weighed in Dow Corning silicone oil contained in a long heavy-walled aluminum cup, which was placed in a vertical position in a $1,000-\mathrm{ml}$ Dewar flask. Weighings were made with a magnetically damped analytical balance. It was found that the specimens could be weighed in the immersion liquid to $\pm 0.5 \mathrm{mg}$ (this includes the effect of temperature variations). For runs made above room temperature, grade DC 710 oil was used. The oil was heated by insulated electrical resistance wire wound around the aluminum cylinder. A thermo-

${ }^{2}$ Optically clear material that is highly crystalline may be obtained by heating the polymer to $250^{\circ} \mathrm{C}$, rapidly quenching to $0^{\circ} \mathrm{C}$, and then carrying out the crystallization for several days at $160^{\circ}$ to $190^{\circ} \mathrm{C}$. Such polymer contains no spherulites.

3 The authors are indebted to L. Mandelkern for making this precise value obtained from the original data available to them 
couple attached to the aluminum cup was used as the sensing element for the $\mathrm{d}-\mathrm{c}$ amplifier, that controlled the bath temperature. The temperature of the sample was determined by using a thermocouple placed within $3 \mathrm{~mm}$ of the sample; this thermocouple was checked against an NBS-calibrated platinum resistance thermometer. It was estimated that the mean temperature of the samples was known to at least $0.2^{\circ} \mathrm{C}$. This figure includes the effect of temperature variations due to fluctuations in the controller, and thermal gradients in the bath (see below). Runs below room temperature were made by surrounding the $1,000-\mathrm{ml}$ Dewar flask with a dry ice-acetone mixture contained in a larger Dewar flask. The less viscous grade DC 200 silicone oil was generally used for the low-temperature studies.

The buoyancy of the silicone oil was calibrated at all temperatures employed in each run by weighing a 5.7038-g piece of transparent and bubble-free fused silica in the oil. The density of the fused silica, as obtained by weighing it in distilled water, was found to be $2.204 \mathrm{~g} \mathrm{~cm}^{-3}$, which is in good agreement with the best values of 2.202 to $2.204 \mathrm{~g} \mathrm{~cm}^{-3}$ given by Sosman [9]. This precaution was taken to show that the material was fused silica, and not crystalline quartz, and also served as a convenient calibration. The specific volume of the samples in $\mathrm{cm}^{3} \mathrm{~g}^{-1}$ was calculated with the formula

$$
\bar{V}_{s}=\frac{W_{\mathrm{SiO}_{2}}(\text { air }) \times\left[W_{s}(\text { air })-W_{s}(\text { bath })\right]}{W_{s}(\text { air }) \times \rho_{\mathrm{SiO}_{2}} \times\left[W_{\mathrm{SiO}_{2}}(\text { air })-W_{\mathrm{SiO}_{2}}(\text { bath })\right]},
$$

where $W_{\mathrm{SiO}_{2}}$ is the mass of the fused silica in the indicated medium (air or oil bath), $W_{s}$ the mass of the polymer sample in the indicated medium, and $\rho_{\mathrm{SiO}_{2}}$ the density of the fused silica. The quantities $W_{s}$ (bath) and $W_{\mathrm{siO}_{2}}$ (bath), refer to the mass as measured in the bath at the temperature of measurement. The coefficient of expansion data of Souder and Hidnert [10] show that the density of fused silica remains within about 4 parts in 10,000 of its room-temperature value over the temperature range of interest here. Therefore, the value $\rho_{\mathrm{SiO}_{2}}$ (air) $=$ $2.204 \mathrm{~g} \mathrm{~cm}^{-3}$ was used at all temperatures. The results for the specific volume of the polymer were occasionally checked between $3^{\circ}$ and $95^{\circ} \mathrm{C}$ by weighing the samples in distilled water.

All of the solid specimens were annealed for a short time at a temperature of $70^{\circ}$ to $90^{\circ} \mathrm{C}$ prior to making a series of measurements in order to relieve any strains in them.

Attention was given to a number of possible sources of error. The silicone oil bath was examined for the presence of thermal gradients of sufficient magnitude to affect the results. By mapping the oil bath with a thermocouple, it was demonstrated that these were always negligibly small (less than $\pm 0.2^{\circ} \mathrm{C}$ ) in the vicinity of the sample. It was determined by weighing a thin metal strip suspended vertically in the bath, and then crimping the strip and repeating the operation, that convection currents, if present, did not affect the measurements by alter- ing the buoyancy. Special attention was also given to the problem of sorption of silicone oil into the samples. At temperatures below $200^{\circ} \mathrm{C}$, the uptake of silicone oil (as measured by weighing the $5-\mathrm{g}$ samples in air) was always less than $0.2 \mathrm{mg}$ even after prolonged immersion. This is obviously insufficient to cause appreciable error. Furthermore, it was found that by making measurements as soon as possible after temperature equilibrium was fully attained ( 7 to $10 \mathrm{~min}$ ), it was feasible to measure the specific volume in the liquid state up to $260^{\circ} \mathrm{C}$ without undue sorption of the oil in the polymer. (The polymer is still viscous enough even at this temperature to remain on the suspension wire for the required tim€.) After a number of runs the liquid polymer tended to inbibe about $10 \mathrm{mg}$ of silicone oil, but even in such cases, results indistinguishable from those on specimens free of silicone were obtained if the original weight of the polymer prior to any sorption was used in the calculations. It is safe to conclude that the results are free of errors due to sorption of silicone oil. Two other effects that warranted consideration were the small downward thrust (about $1 \mathrm{mg}$ ) on the suspension wire due to surface tension at the silicone-oil-air interface, and the buoyant effect of the oil on that part of the wire in the bath. It was found that these two effects compensated one another over a wide range of temperature if a $12.5-\mathrm{cm}$ length of the Chromel suspension wire was allowed to remain in the oil. The results obtained by using this procedure closely checked those obtained by using the usual but more cumbersome methods. Care was taken to remove any small deposits of silicone oil that might form on the suspension wire. Finally, no effects attributable to degradation were found. Under the conditions described, i. e., prolonged crystallization in nitrogen followed by measurement in silicone oil, the samples never exhibited any significant loss of weight or discoloration on heating, no bubbles were ever observed to form in them, and samples repeatedly heated to high temperatures always gave substantially the same results as fresh material.

The reproducibility of specific-volume measurements on any particular sample measured in silicone oil in the range of $0^{\circ}$ to $120^{\circ} \mathrm{C}$ was $\pm 0.0001 \mathrm{~cm}^{3} \mathrm{~g}^{-1}$. The reproducibility was somewhat poorer at the extremes of the temperature range covered, but never exceeded $\pm 0.0004 \mathrm{~cm}^{3} \mathrm{~g}^{-1}$, except perhaps in the range between $190^{\circ}$ and $216^{\circ} \mathrm{C}$, where rapid melting took place. A comparison of the results for the same sample measured in distilled water, and then in silicone oil at the same temperature, usually showed agreement to within $0.0003 \mathrm{~cm}^{3} \mathrm{~g}^{-1}$. It is estimated from the latter figure, by taking the density of distilled water as the appropriate reference standard, that the specific volume values quoted for the polymer are accurate to at least 1 part in 1,000 .

\section{Results}

The specific-volume values obtained for the quenched and crystallized samples are plotted in figure 1 as a function of temperature. The 


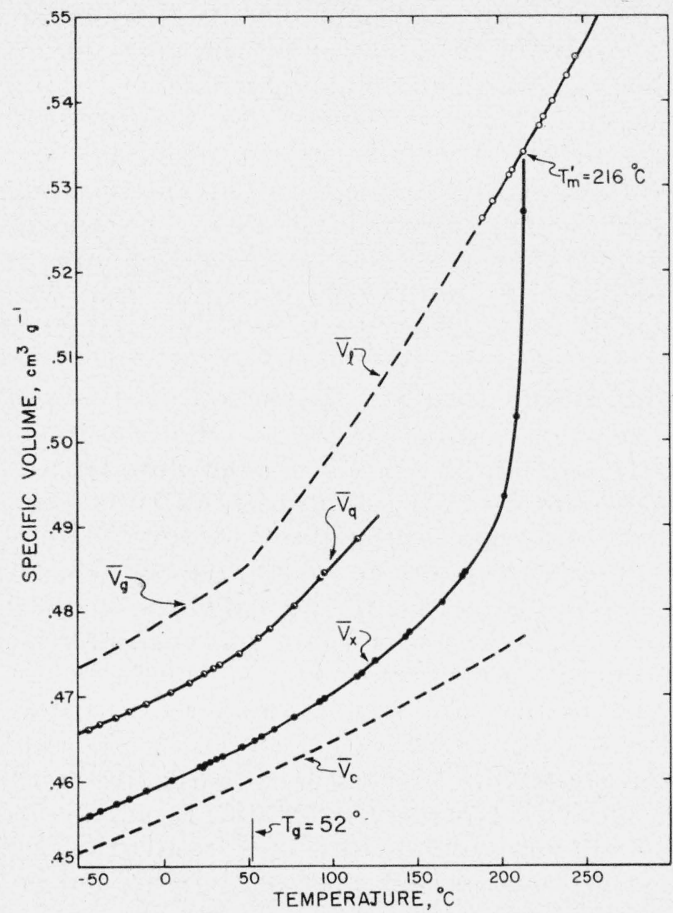

FIGURE 1. Specific volume of poly (chlorotrifuoroethylene) as a function of temperature.

, experimental points for 5-day crystallized specimens $(x \sim 12)$, experimental points for quenched specimen ( 12 X) . experimental points for pure liquid and supercooled $\sim 12 \times, O$, experimental points for pure liquid and supercooled umes of pure glassy, crystalline, and highly supercooled phases

symbol $\bar{V}_{x}$ is used to denote the specific volume of the 5-day crystallized sample, and $\bar{V}_{q}$ is used to denote the corresponding quantity for the quenched sample. A number of the single points shown in figure 1 actually represent duplicate determinations that could not be plotted distinctly owing to the scale used in the diagram.

Values of $\bar{V}_{x}$ and $\bar{V}_{q}$ corresponding to the best line drawn through the data when plotted on a large scale are given in table 1 in boldfaced type (the numbers in ordinary type are derived quantities). This operation was carried out with special care in the range $-40^{\circ}$ to $+115^{\circ} \mathrm{C}$ for both specimens, because it is in this region that volume-temperature derivatives must be known with good precision. In this temperature interval, the standard deviation of the experimental points from the best line was $0.0001 \mathrm{~cm}^{3} \mathrm{~g}^{-1}$.

The 5-day sample is sufficiently stable to permit highly reproducible specific-volume-temperature data to be obtained. It was found that the $\bar{V}_{x}$ value at a given temperature anywhere in the range $-40^{\circ}$ to $+205^{\circ} \mathrm{C}$ did not change in a period of 30 days; the specific-volume curve is essentially completely reversible if measured within this time interval. It will be shown subsequently that this specimen is about 82 percent crystalline. It is probable that it is in a quasi-equilibrium state with respect to the degree of crystallinity, though it may not be too far removed from the true equilibrium state. Thus, the apparent cessation of the crystallization process lead- ing to the stability of the 5-day specimen may well be largely a result of the extreme slowness of the crystallization process characteristic of the highly crystalline material [3].

The melting point of the 5-day crystallized material is referred to as the quasi-equilibrium melting point, $T_{m}^{\prime}$. This is defined experimentally as the temperature where the $\bar{V}_{x}$ and $\bar{V}_{z}$ curves intersect (fig. 1). For specimens crystallized in the manner described, $T_{m}^{\prime}=216^{\circ} \mathrm{C}$. The equilibrium melting temperature, $T_{m}$, which is the one of thermodynamic significance [11], is certainly somewhat higher. Samples in which unstrained crystals melt at $218.0^{\circ} \mathrm{C}$ have already been prepared, and other lines of evidence $[3,12]$ suggest that $T_{m}$ lies in the range $220^{\circ}$ to $225^{\circ} \mathrm{C}$. For the time being, we have indicated the nominal value $T_{m} \cong 220^{\circ} \mathrm{C}$ in table 1 .

TABLE 1. Specific volume of poly-(chlorotrifluoroethylene) as a function of temperature a

\begin{tabular}{|c|c|c|c|c|}
\hline Temperature & $\begin{array}{l}\text { Crystallized } \\
\text { sample (5 } \\
\text { day), } \bar{V}_{x}\end{array}$ & $\begin{array}{l}\text { Quenched } \\
\text { sample, } \\
\overline{\bar{V}}_{a}\end{array}$ & $\begin{array}{l}\text { Pure crys- } \\
\text { tal, } \bar{V}_{c}\end{array}$ & $\begin{array}{c}\text { Pure liquid, } \\
\text { supercooled } \\
\text { liquid, or glass, b } \\
\bar{V}_{l, g}\end{array}$ \\
\hline $\begin{array}{l}{ }^{\circ} C \\
-40 \\
-20 \\
0 \\
+20 \\
52\left(T_{g}\right)\end{array}$ & $\begin{array}{r}0.45643 \\
.45805 \\
.45975 \\
.46156 \\
.46466\end{array}$ & $\begin{array}{r}0.46595 \\
.46786 \\
.46992 \\
.47213 \\
.47635\end{array}$ & $\begin{array}{r}0.45254 \\
.45404 \\
.45563 \\
.45728 \\
.46007\end{array}$ & $\left.\begin{array}{r}0.47445 \\
.47659 \\
.47884 \\
.48138 \\
.48560\end{array}\right\} \bar{V}_{g}$ \\
\hline $\begin{array}{r}65 \\
80 \\
100 \\
115 \\
130\end{array}$ & $\begin{array}{l}.46618 \\
.46803 \\
.47057 \\
.47256 \\
.4747\end{array}$ & $\begin{array}{l}.47824 \\
.48119 \\
.48526 \\
.48841 \\
-. .-.-.\end{array}$ & $\begin{array}{l}.46125 \\
.46265 \\
. \mathbf{4 6 4 5 8} \\
. \mathbf{4 6 6 0 0} \\
. \mathbf{4 6 7 6 1}\end{array}$ & $\begin{array}{l}.48890 \\
.49284 \\
.49830 \\
.50255 \\
.50693\end{array}$ \\
\hline $\begin{array}{l}150 \\
180 \\
190 \\
200 \\
210\end{array}$ & $\begin{array}{l}.4780 \\
.4847 \\
.4881 \\
.4928 \\
.5027\end{array}$ & 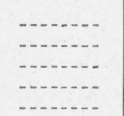 & $\begin{array}{l}.46972 \\
.47300 \\
.47414 \\
.47529 \\
.47645\end{array}$ & $\left.\begin{array}{l}.51297 \\
.52249 \\
.52585 \\
.52915 \\
.53253\end{array}\right\} \bar{V}_{l}$ \\
\hline $\begin{array}{l}215 \\
216\left(T_{m}^{\prime}\right) \\
220\left(T_{m}^{\prime}\right)^{\circ} \\
225 \\
240\end{array}$ & $\begin{array}{l}.5270 \\
.5346 \\
-.0 \\
-0 .-\end{array}$ & - & $\begin{array}{l}.47704 \\
.47716 \\
.47763 \\
. . .- \\
. .2\end{array}$ & $\begin{array}{r}.53425 \\
.53459 \\
.53598 \\
.53774 \\
.54310\end{array}$ \\
\hline 260 & $\ldots$ & - n & - n & .55050 \\
\hline
\end{tabular}

a Boldfaced numbers represent smoothed values of specific volume obtained from actual experimental data. Numbers in ordinary type are derived quantities. All specific volumes are in $\mathrm{cm}^{3} \mathrm{~g}-1$.

${ }_{b}$ The symbol $\bar{V}_{3}$ is used to denote the specific volume of both the liquid and supercooled liquid; $\bar{V}_{g}$ represents the specific volume of the glassy state.

A Approximate value.

The quenched sample is in a metastable state below about $115^{\circ} \mathrm{C}$. Above this temperature, crystallization begins to take place at a perceptible rate, and the specific volume tends toward that found for the crystallized specimen. So long as the residence time in the temperature range $110^{\circ}$ to $120^{\circ} \mathrm{C}$ is kept short, the $\bar{V}_{q}$ curve shown in figure 1 is reversible (cf [8]). It will be shown that the quenched specimen is about 39 percent crystalline.

Specific-volume data for the liquid and supercooled liquid polymer are also plotted in figure 1 , the symbol $\bar{V}_{l}$ being used to denote both of these phases. Values were obtained in the supercooled region by cooling the samples from the melt to the indicated temperature, and making the measurements prior to the onset of any appreciable amount 
of crystallization It was found that the crystallization became too rapid to permit reliable data to be obtained when the temperature fell below $190^{\circ}$ C. ${ }^{4}$ The specific-volume data for the liquid and supercooled region from $260^{\circ}$ to $190^{\circ} \mathrm{C}, 20$ experimental points in all, were fitted by using least-squares to an equation of the form $\bar{V}_{l}=\alpha+\beta T+\gamma T^{2}$, using an electronic computer. ${ }^{5}$ Values of the specific voulme obtained from this analysis are given at selected temperatures in table 1 in boldfaced type. The standard deviation in this region proved to be $0.00037 \mathrm{~cm}^{3} \mathrm{~g}^{-1}$.

The method that will be used to calculate the degree of crystallinity makes use of the volumetemperature derivatives of the quenched and crystalline samples as they would exist at or near $T_{g}$. The volume-temperature derivatives, ${ }^{6} d \bar{V} / d T$, of the crystalline, quenched, liquid, and supercooled liquid polymer are plotted as a function of temperature in figure 2. These data were obtained by using the information listed in table 1 . In the region $-40^{\circ}$ to

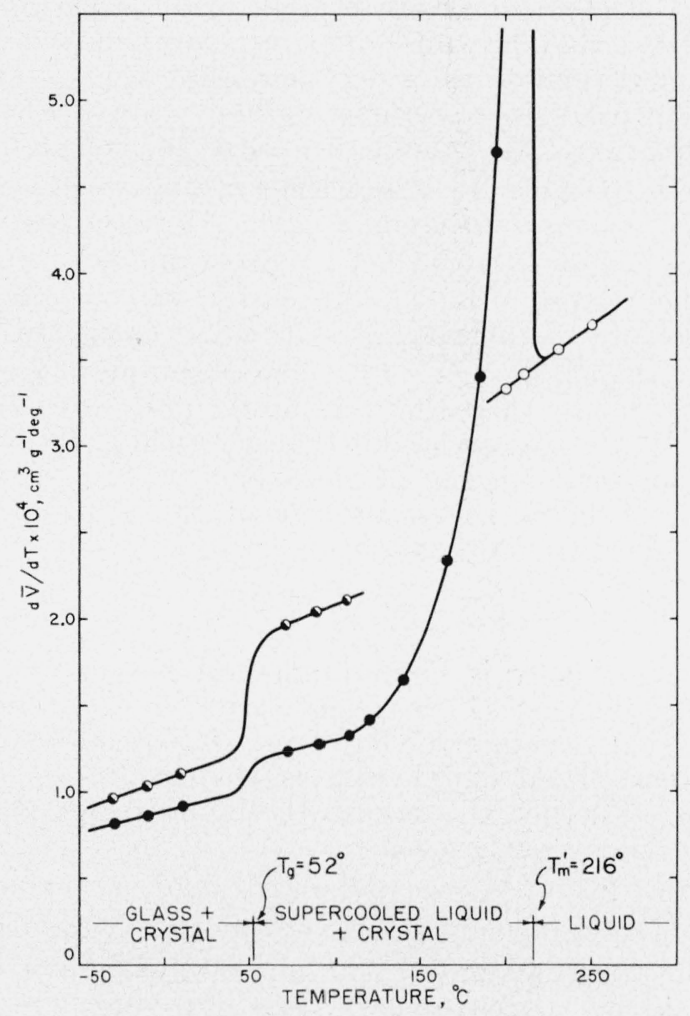

Figure 2. Volume-temperature derivatives of the poly(chlorotrifluoroethylene) specimens as a function of temperature.

, 5-day crystallized material; $\mathbf{1}$, quenched material; $\bigcirc$, liquid and supercooled liquid material.

4. Considerable care must be exercised to prevent crystallization from affecting the precision of the data in the supercooled region. Brief heat treatment at ca. $300^{\circ} \mathrm{C}$ renders almost all of the heterogeneous nuclei in the polymer inactive, and thus lowers the rate of crystallization in the supercooled region. This permitted precise data to be obtained down to $190^{\circ} \mathrm{C}$ in the present case. It was found that accurate results could not be obtained at lower temperatures by extrapolating back to zero time.

5 The authors thank J. M. Cameron of the statistical engineering laboratory of NBS for carrying out this analysis.

${ }^{6}$ Strictly speaking, $d \bar{V} / d T$ is the specific-volume-temperature derivative, but this cumbersome term is not used in the text. $+115^{\circ} \mathrm{C}$, an analysis of the volume-temperature derivatives, as calculated from the original experimental points, shows that the standard deviation is a little less than 3 percent in this interval. The smoothed data in table 1 lead to $d \bar{V} / d T$ versus $T$ curves that are for all practical purposes identical to those obtained from the original experimental points.

It is seen in figure 2 that there is no upswing in the volume-temperature derivative of the crystallized sample indicative of melting out of small crystallites until a temperature of about $120^{\circ} \mathrm{C}$ is reached. This, together with the fact that the specific volume of both the quenched and crystallized specimens are invariant with time below $\sim 115^{\circ}$ $\mathrm{C}$, justifies the assumption to be used later that the degree of crystallinity of the specimens does not change appreciably below $115^{\circ} \mathrm{C}$.

A glass transition is clearly apparent in the $d \bar{V} / d T$ data for the quenched sample between $40^{\circ}$ and $60^{\circ} \mathrm{C}$ (fig. 2). A similar but less obvious transition also appears in the crystallized specimen, a fact which conclusively shows that it is not completely crystallized. The glass transition takes place only in the amorphous component of the polymer. Hence the semicrystalline samples consist of glass plus crystal below $T_{g}$, and supercooled liquid plus crystal from $T_{g}$ up to $T_{m}^{\prime}$.

The glass-transition temperature has been determined by plotting the difference between the specific volume of the crystalline and quenched samples as a function of temperature by using the smoothed data in table 1 (fig. 3). This method tends to sub-

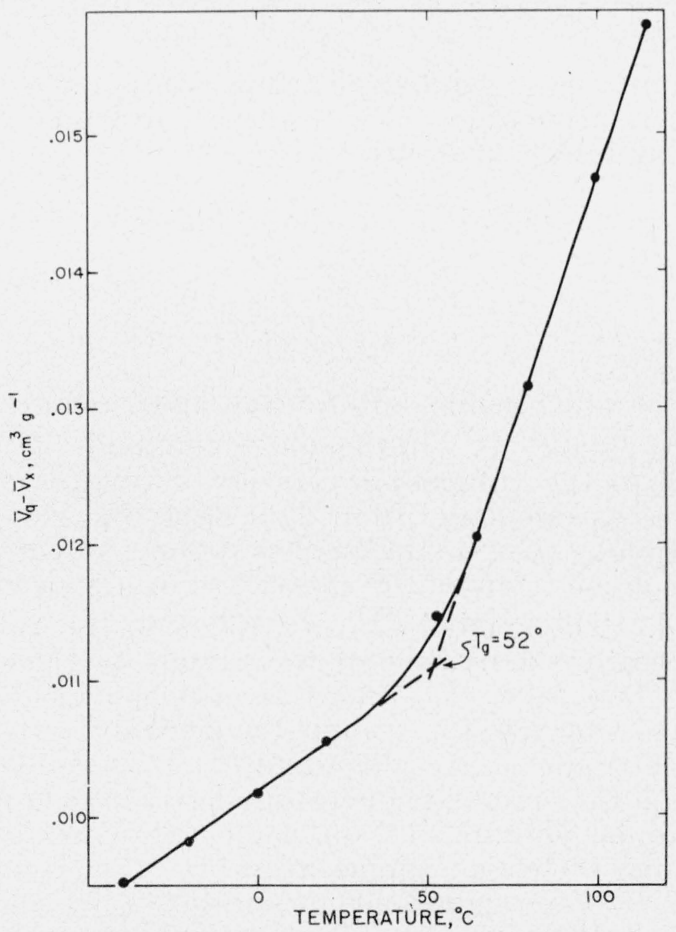

FIguRE 3. Determination of the glass-transition temperature, $T_{\boldsymbol{g}}$, for poly(chlorotrifluoroethylene). 
tract out the greater part of the curvature inherent in the volume-temperature curves, and therefore yields a plot through which two intersecting straight lines may be drawn with some confidence. The glass-transition temperature determined in this way is $52^{\circ} \mathrm{C}$. This is in fair agreement with the value of $45^{\circ} \mathrm{C}$ determined by Mandelkern and co-workers [8], and a value attributed to Reding [13], and is in good agreement with the value of $50^{\circ} \mathrm{C}$ mentioned by Boyer [14]. A further discussion of the glass transition is given in section 6.5.

\section{Theory}

\subsection{Preliminary Considerations}

The basic assumption is made that the volumes of the crystalline and amorphous material in a semicrystalline polymer are additive. In the units used here, namely, specific volume, this leads to the relation

$$
\chi_{s} \bar{V}_{c}+\left(1-\chi_{s}\right) \bar{V}_{l, g}=\bar{V}_{s}
$$

where $\bar{V}_{l, g}$ is the specific volume of the purely amorphous (supercooled liquid or glassy) regions, $\bar{V}_{s}$ is the specific volume of the semicrystalline sample, and $\bar{V}_{c}$ the specific volume of the entirely crystalline material. $\chi_{s}$ is the mass fraction of the sample, that is crystalline, and will! henceforth be called the degree of crystallinity. ${ }^{7}$ It is seen from eq (1) that

$$
\chi_{s}=\frac{\bar{V}_{l, g}-\bar{V}_{s}}{\bar{V}_{l, g}-\bar{V}_{c}}
$$

This formula is valid at all temperatures. By taking the derivative of eq (1) with respect to temperature, we obtain the equations

$$
\chi_{s}\left(d \bar{V}_{c} / d T\right)+\left(1-\chi_{s}\right)\left(d \bar{V}_{l} / d T\right)=\left(d \bar{V}_{s}^{+} / d T\right)
$$

and

$$
\chi_{s}\left(d \overline{V_{c}} / d T\right)+\left(1-\chi_{s}\right)\left(d \overline{V_{g}} / d T\right)=\left(d \overline{V_{s}^{-}} / d T\right),
$$

each of which holds only for any given temperature where $d \chi_{s} / d T=0 . \quad$ In the above equations, $d \bar{V}_{s}^{+} / d T$ refers to the volume-temperature derivative of the sample as calculated from data above $T_{g}$, where the amorphous part of the semicrystalline polymer has the volume-temperature derivative of a supercooled liquid. The symbol $d \bar{V}_{s}^{-} / d T$ refers to the volumetemperature derivative of the sample as calculated from data below $T_{g}$, where the amorphous part of the polymer has the volume-temperature derivative characteristic of the glassy state. It is understood that all the volume-temperature derivatives in eq (3) are to be obtained at the same temperature, and a similar statement applies to eq (4). The condition $d \chi_{s} / d T=0$ certainly holds at and below $T_{g}$, and can generally be shown to apply at temperatures sufficiently far above $T_{g}$ to permit the estimation of

\footnotetext{
${ }^{7}$ The volume fraction of crystals is given by the expression $\lambda_{s}=\left(\rho_{s}-\rho_{l, g}\right) /\left(\rho_{c}-\rho_{l, g}\right)$,
where $\rho$ is the density.
}

$d \bar{V}_{s}^{+} / d T$. From eq (3) and (4) we obtain the relations

$$
\chi_{s}=\frac{\left(d \bar{V}_{l} / d T\right)-\left(d \bar{V}_{s}^{+} / d T\right)}{\left(d \bar{V}_{l} / d T\right)-\left(d \bar{V}_{c} / d T\right)}
$$

and

$$
\chi_{s}=\frac{\left(d \bar{V}_{g} / d T\right)-\left(d \bar{V}_{s}^{-} / d T\right)}{\left(d \bar{V}_{g} / d T\right)-\left(d \bar{V}_{c} / d T\right)} .
$$

For any temperatures where $d \chi_{s} / d T=0$, eq (2), (5), and (6) must lead to identical values of $\chi_{s}$ for a given sample.

It is instructive at this point to indicate the nature of the difficulties that are encountered in estimating the degree of crystallinity from specificvolume measurements alone if data on only a single specimen are used. It is assumed in the following discussion that specific-volume data exist for the semicrystalline range from well below $T_{g}$ up to $T_{m}^{\prime}$, and for the liquid range near and well above $T_{m}^{\prime}$. These circumstances correspond to the experimental situation that prevails for poly (chlorotrifluoroethylene) and certain other polymers that tend to transform rapidly to a semicrystalline state below $T_{m}^{\prime}$.

Equation (2) cannot be used to calculate $\chi_{s}$ directly because $\bar{V}_{c}$ is not known, and we therefore turn to a consideration of eq (5). Here the quantity $d \bar{V}_{s}^{+} / d T$ may be regarded as experimentally known in the region near $T_{g}$. In order to compute the degree of crystallinity, it is thus necessary to estimate $d \bar{V}_{l} / d T$ and $d \vec{V}_{c} / d T$. The assumption is commonly made that the volume-temperature derivative of the semicrystalline sample below $T_{g}$, where the polymer consists of glass plus crystal, may be taken as being representative of the pure crystal. This gives the approximation

$$
d \bar{V}_{s}-d T \cong d \bar{V}_{c} / d T .
$$

However, there is no fundamental reason for supposing that eq (7) is really exact (it will emerge later that it is in error by about 10 percent even for the crystallized sample of poly(chlorotrifluroroethylene)). Further, the data in the liquid region can be analyzed by using a least-squares fit to an equation of the form $\bar{V}_{l}=\alpha+\beta T+\gamma T^{2}$, and a value of $d \bar{V}_{l} / d T$ obtained by a long downward extrapolation to the range where $d \bar{V}_{c} / d T$ and $d \bar{V}_{s}^{+} / d T$ have been evaluated. Unfortunately, this procedure will generally lead to a rather uncertain $d \bar{V}_{l} / d T$ value unless the specific-volume data for the liquid are extraordinarily precise. In the case of poly(chlorotrifluoroethylene), where the standard deviation of the $\bar{V}_{l}$ equation in the liquid range is only $0.00037 \mathrm{~cm}^{3} \mathrm{~g}^{-1}$, the $d \bar{V}_{l} / d T$ value at $T_{g}$ as obtained by the extrapolation procedure is $2.27 \times 10^{-4} \mathrm{~cm}^{3} g^{-1} \mathrm{deg}^{-1}$, with a standard deviation of $0.80 \times 10^{-4}$. This corresponds to a coefficient of variation of 35 percent. It is clear from the foregoing that the use of approximation (7), together with the long extrapolation necessary to estimate $d \bar{V}_{l} / d T$ at or near $T_{g}$, will 
tend to produce large errors in $\chi_{s}$ despite the precision of the input data in the region of direct measure ment.

The approximate nature of the procedures required to analyze data on a single specimen also have an adverse effect on the accuracy of $\bar{V}_{l}$ and $\bar{V}_{c}$ at $T_{g}$. By using a long downward extrapolation of the liquid data, a rough value of $\bar{V}_{l}$ can be obtained at $T_{g}$. For poly-(chlorotrifluoroethylene), $\bar{V}_{l}$ turns out to be $0.4875 \mathrm{~cm}^{3} \mathrm{~g}^{-1}$, with a standard deviation of 0.0070 , despite the relatively high precision of the input data in the region of measurement (the standard deviation would have been even greater had the equation $\bar{V}_{l}=\alpha+\beta T$ been used to represent the liquid data). If this quantity is inserted in eq (2), together with the rough value of $\chi_{s}$ previously obtained, an estimate of $\bar{V}_{c}$ at $T_{g}$ can be made. It is clear, however, that crystal properties determined in this way are not especially reliable.

It is seen that the major difficulty with the singlespecimen method is the inaccuracy of the values of $\bar{V}_{l}$ and $d \bar{V}_{l} / d T$ at $T_{\mathrm{g}}$ obtained by the long "unguided" extrapolation of the liquid data from high temperatures. In order to obtain satisfactory results it would be necessary to know $\bar{V}_{l}$ at $T_{g}$ to within about $0.0015 \mathrm{~cm}^{3} \mathrm{~g}^{-1}$, and $d \bar{V}_{l} / d T$ at the same temperature to within about 5 percent. In the present case, this would require data in the experimentally accessible liquid range $\left(190^{\circ}\right.$ to $\left.260^{\circ} \mathrm{C}\right)$, which had a standard deviation of about $0.00007 \mathrm{~cm}^{3} \mathrm{~g}^{-1}$. Data of such high precision would not be easily obtained for any polymer in the liquid state, especially at high temperatures.

In this type of situation, it is considerably more satisfactory to employ the method to be described in section 4.2, which deals with two samples, one highly crystalline and the other as amorphous as is practical. This method completely avoids the use of approximation (7), and does not require extrapolation over long temperature ranges. The principal input data are obtained where the measurements are most precise, and the volume-temperature derivatives are obtained at a common temperature. Both $\bar{V}_{l}$ and $\bar{V}_{c}$ can be obtained to within about 0.0015 $\mathrm{cm}^{3} \mathrm{~g}^{-1}$, and the degree of crystallinity to a standard deviation of about 0.05 .

\subsection{Calculation of the Degree of Crystallinity Near $\mathrm{T}_{g}$ Using Two specimens}

Consider now the case of two samples, one highly crystalline and the other strongly quenched. The degree of crystallinity of these samples is denoted $\chi_{x}$ and $\chi_{q}$, respectively. Thus we may write

$$
\chi_{q}=\frac{\bar{V}_{l}-\bar{V}_{q}}{\bar{V}_{l}-\bar{V}_{c}}=\frac{\left(d \bar{V}_{l} / d T\right)-\left(d \bar{V}_{q}^{+} / d T\right)}{\left(d \bar{V}_{l} / d T\right)-\left(d \bar{V}_{c} / d T\right)},
$$

and

$$
\chi_{x}=\frac{\bar{V}_{l}-\bar{V}_{x}}{\bar{V}_{l}-\bar{V}_{c}}=\frac{\left(d \bar{V}_{l} / d T\right)-\left(d \bar{V}_{x}^{+} / d T\right)}{\left(d \bar{V}_{l} / d T\right)-\left(d \bar{V}_{c} / d T\right)}
$$

by analogy with eq (2) and (5). The quantities $\bar{V}_{q}, \bar{V}_{x}, d \bar{V}_{q}^{+} / d T$, and $d \bar{V}_{x}^{+} / d T$ are known and the quantities $\chi_{l}, \chi_{x}, \bar{V}_{l}, \bar{V}_{c}$, and $d \bar{V}_{l} / d T$ are unknown. Equations (8) and (9) contain the important notion that, for a given specimen, the degree of crystallinity computed directly from the specific volumes must be identical to that obtained from the volume-temperature derivatives when $d \chi / d T=0$. Initially the degree of crvstallinity will be calculated from data in the vicinity of the glass transition where it is certain that the condition $d \chi / d T=0$ is fulfilled.

The first step in the procedure is to construct an equation for $\bar{V}_{l}$ that gives an acceptable fit in the liquid region and conforms with the demands imposed by eq (8) and (9). An important assumption at this point is that the correct form of the equation for the liquid and supercooled liquid all the way down to $T_{g}$ is

$$
\bar{V}_{l}=A+B T+C T^{2} .
$$

By eliminating the ratio $\left[\left(d \bar{V}_{l} / d T\right)-\left(d \bar{V}_{c} / d T\right)\right] /$ $\left(\bar{V}_{l}-\bar{V}_{c}\right)$ from eq $(8)$ and $(9)$, one finds

$$
\begin{aligned}
& \bar{V}_{l}\left[\left(d \bar{V}_{q}^{+} / d T\right)-\left(d \bar{V}_{x}^{+} / d T\right)\right]+\left(d \bar{V}_{l} / d T\right)\left(\bar{V}_{x}-\bar{V}_{q}\right) \\
= & \bar{V}_{x}\left(d \bar{V}_{q}^{+} / d T\right)-\bar{V}_{q}\left(d \bar{V}_{x}^{+} / d T\right) .
\end{aligned}
$$

Then substituting $\bar{V}_{l}=A+B T+C T^{2}$ and $d \bar{V}_{l} / d T=$ $B+2 C T$ into this equation, the relation

$$
\begin{aligned}
& A\left[\left(d \bar{V}_{q}^{+} / d T\right)-\left(d \bar{V}_{x}^{+} / d T\right)\right]+ \\
& B\left\{\left(\bar{V}_{x}-\bar{V}_{q}\right)+T\left[\left(d \bar{V}_{q}^{+} / d T\right)-\left(d \bar{V}_{x}^{+} / d T\right)\right]\right\}+ \\
& C\left\{\left[\left(d \bar{V}_{q}^{+} / d T\right)-\left(d \bar{V}_{x}^{+} / d T\right)\right] T^{2}+2 T\left(\bar{V}_{x}-\bar{V}_{q}\right)\right\} \\
= & \bar{V}_{x}\left(d \bar{V}_{q}^{+} / d T\right)-\bar{V}_{q}\left(d \bar{V}_{x}^{+} / d T\right)
\end{aligned}
$$

is obtained. The volume-temperature derivatives in eq (12) must, of course, all be obtained at some common temperature. It is convenient, though not necessary, to choose $T=T_{\mathrm{g}}$. Observe that all of the quantities in eq (12), except $A, B$, and $C$, are experimentally known from the specific-volume and volume-temperature derivatives as obtained on the two semicrystalline specimens in the vicinity of $T_{\mathrm{g}}$. At this point, two equations describing the liquid data at two temperatures, $T_{1}$ and $T_{2}$, in the region of direct measurement are introduced:

$$
\begin{aligned}
& A+B T_{1}+C T_{1}^{2}=\bar{V}_{l}{ }^{\left(T_{1}\right)} \\
& A+B T_{2}+C T_{2}^{2}=\bar{V}_{l}{ }^{\left(T_{2}\right)} .
\end{aligned}
$$

Since $\bar{V}_{l}$ is known experimentally at $T_{1}$ and $T_{2}$, the three simultaneous equations (12 to 14 ) may now be solved for $A, B$, and $C$. This yields an equation for the liquidus down to $T_{z}$, which not only fits the observed liquid data extremely well, but also gives $\bar{V}_{l}$ and $d \bar{V}_{l} / d T$ values near $T_{g}$, which, because of the conditions imposed by eq (8) and (9), are certain to yield the same degree of crystallinity for a given 
sample by both the specific-volume and volumetemperature derivative methods. The values of $\bar{V}_{l}$ and $d \bar{V}_{l} / d T$ at $T_{g}$ are, through eq (12), controlled largely by the experimental volume-temperature derivative data on the semicrystalline samples in the general vicinity of $T_{g}$, and not by a long extrapolation of the observed liquid data down to $T_{\mathrm{g}}$. The fundamental nature of the method is most easily perceived by noting that eq (12) is altogether equivalent to having another equation of the form of (13) and (14) where $V_{l}$ is known at $T_{g}$.

The next step involves the calculation of the volume-temperature derivative of the pure crystal. By eliminating the ratio $\left(d \bar{V}_{l} / d T-d \bar{V}_{c} / d T\right) /\left(\overline{d V}_{g}\right)$ $\left.d T-d \bar{V}_{c} / d T\right)$ from the equations

$$
\chi_{q}=\frac{\left(d \bar{V}_{g} / d T\right)-\left(d \bar{V}_{q}^{-} / d T\right)}{\left(d \bar{V}_{g} / d T\right)-\left(d \bar{V}_{c} / d T\right)}=\frac{\left(d \bar{V}_{l} / d T\right)-\left(d \bar{V}_{q}^{+} / d T\right)}{\left(d \bar{V}_{l} / d T\right)-\left(d \bar{V}_{c} / d T\right)},
$$

and

$$
\chi_{x}=\frac{\left(d \bar{V}_{g} / d T\right)-\left(d \bar{V}_{x}^{-} / d T\right)}{\left(d \bar{V}_{g} / d T\right)-\left(d \bar{V}_{c} / d T\right)}=\frac{\left(d \bar{V}_{l} / d T\right)-\left(d \bar{V}_{x}^{+} / d T\right)}{\left(d \bar{V}_{l} / d T\right)-\left(d \bar{V}_{c} / d T\right)},
$$

which are based on eq (5) and (6), and then eliminating $d \bar{V}_{g} / d T$, it is found that

$d \bar{V}_{c} / d T=$

$$
\frac{\left(d \bar{V}_{x}^{+} / d T\right)\left(d \bar{V}_{q}^{-} / d T\right)-\left(d \bar{V}_{x}^{-} / d T\right)\left(d \bar{V}_{q}^{+} / d T\right)}{\left(d \bar{V}_{x}^{+} / d T\right)+\left(d \bar{V}_{q}^{-} / d T\right)-\left(d \bar{V}_{x}^{-} / d T\right)-\left(d \bar{V}_{q}^{+} / d T\right.} .
$$

The term on the right-hand side of the expression involves only experimentally known quantities. The degree of crystallinity of each sample is then computed by inserting the known values of $d \bar{V}_{c} / d T$, $d \bar{V}_{l} / d T, d \bar{V}_{x}^{+} d T$, and $d \bar{V}_{q}^{+} / d T$ into eq (8) and (9). As a final step, $\bar{V}_{c}$ may be calculated from both eq (8) and (9) because $\bar{V}_{l}, \bar{V}_{x}, \bar{V}_{q}$, and $\chi_{x}$ and $\chi_{q}$, are now known. Agreement between the two $\bar{V}_{c}$ values provides a convenient check of the calculations.

\subsection{Calculation of the Degree of Crystallinity as a Function of Temperature}

In order to determine the degree of crystallinity as a function of temperature, an equation of the form of (2) will be used. The specific volume of the sample, $\bar{V}_{s}$, is experimentally known at all temperatures of interest, and $\bar{V}_{l}$ is also determined as a function of temperature by the procedure outlined in the previous section. Hence, if the temperature dependence of $\bar{V}_{c}$ is known, $\chi_{s}$ can then be computed as a function of temperature. The remainder of this section is devoted to showing how the temperature variation of $\bar{V}_{c}$ is determined.

A direct way of obtaining $\bar{V}_{c}$ as a function of temperature, which also yields other information of interest, is to calculate $\bar{V}_{c}$ values above and below $T_{g}$, combine them with that already calculated at $T_{g}$, and solve the three simultaneous equations to obtain the constants in the relation

$$
\bar{V}_{c}=A^{\prime}+B^{\prime} T+C^{\prime} T^{2} .
$$

$\bar{V}_{c}$ can be calculated for that region above $T_{g}$ where $\chi_{x}$ is already evaluated from the relation

$$
\chi_{x}=\frac{\bar{V}_{l}-\bar{V}_{x}}{\bar{V}_{l}-\bar{V}_{c}}
$$

because both $\bar{V}_{\imath}^{\prime}$ and $\bar{V}_{x}$ are known as a function of temperature. (At this stage, $\chi$ is known only at temperatures where $d x / d T=0.) \quad \bar{V}_{c}$ and $\bar{V}_{g}$ can readily be obtained at temperatures below $T_{g}$ by simultaneously solving the equations

and

$$
\chi_{x}=\frac{\overline{V_{g}}-\overline{V_{x}}}{\overline{V_{g}}-\bar{V}_{c}}
$$

$$
\chi_{q}=\frac{\bar{V}_{g}-\bar{V}_{q}}{\bar{V}_{g}-\bar{V}_{c}}
$$

It should be noted that $\bar{V}_{c}$ is actually determined from the data only from the lowest temperature of measurement up to the highest temperature where one is still certain that the degree of crystallinity is not changing with temperature. Thus, to obtain the degree of crystallinity as a function of temperature up to the melting point, an extrapolation of $V_{c}$ is required. Fortunately, this extrapolation will not ordinarily lead to serious errors, since the constants $B^{\prime}$ and $C^{\prime}$ are quite small for a crystal, and can be based on data obtained for a temperature range that is longer than the implied extrapolation. This extrapolation will generally be a much less serious matter than the long "unguided" extrapolation of the liquidus down to $T_{\mathrm{g}}$ discussed in section 4.1 ; for liquids, the temperature range where data can be obtained will for various reasons generally be considerably shorter than the implied extrapolation, and $\beta$ and $\gamma$ will be large owing to the strong dependence of the volume on temperature characteristic of liquids. The method described to obtain $\bar{V}_{c}$ as a function of temperature applies only if there is no solid-state phase transitition in the crystalline material between $T_{g}$ and $T_{m}$.

\section{Application to Poly(chlorotrifluoroethyl- ene)}

\subsection{Degree of Crystallinity Near $\mathrm{T}_{g}$}

The general procedure described in section 4.2 is used here.

The method of deriving the volume-temperature derivatives at $T_{g}$ is illustrated in figure 4 . The volume-temperature derivatives were first calculated at a number of temperatures by using the smoothed 


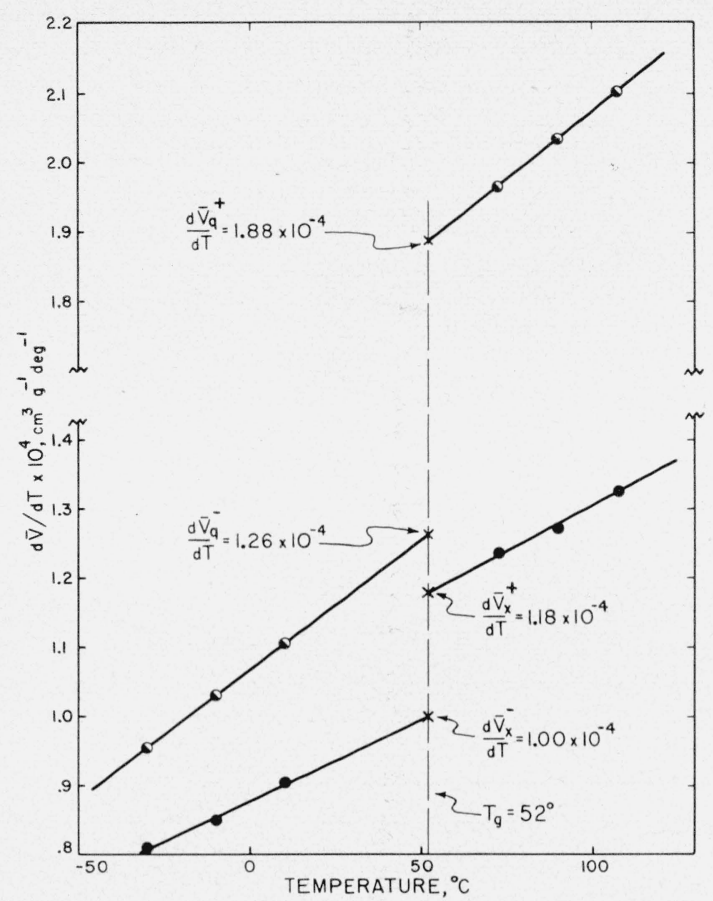

Figure 4. Determination of the volume-temperature derivatives of the 5-day crystallized (-) and quenched (J) specimens at $T_{\mathrm{g}}$,

data in table 1, plotted as a function of temperature, and the desired derivatives obtained at $T_{\mathrm{g}}$ by a short extrapolation. The input data are summarized in table 2. It should be noted that the $\bar{V}_{x}$ and $\bar{V}_{q}$ values at $T_{g}$ listed in table 2 are those which would be obtained if the glass transition occurred abruptly at $52^{\circ} \mathrm{C}$, i. e., at the intersection of two curved lines. The $\bar{V}_{x}$ and $\bar{V}_{q}$ values listed in table 2 are the correct ones to use in the analysis, but final results differing only slightly from those to be given would emerge if those listed in table 1 were used instead.

The equation for the liquid and supercooled liquid is readily calculated. Inserting the data from table 2 into eq (12), one finds

$$
0.700 A-74.60 B-9,651.2 C=0.31212 .
$$

Now, selecting two suitably spaced temperatures in the liquid region, say $210^{\circ}$ and $240^{\circ} \mathrm{C}$, and taking the appropriate specific volumes from table 1, we find:

$$
\begin{aligned}
& A+210 B+44,100 C=0.53253 \\
& A+240 B+57,600 C=0.54310 .
\end{aligned}
$$

Solving these simultaneous equations, it is found that

$$
\bar{V}_{l}=0.47337+2.199 \times 10^{-4} T+2.943 \times 10^{-7} T^{2} .
$$

This equation fits the original liquid data extremely well, the standard deviation being only $0.00040 \mathrm{~cm}^{3} \mathrm{~g}^{-1}$. The calculated value of $\bar{V}_{l}$ at $T_{g}$ is $0.4856 \mathrm{~cm}^{3} \mathrm{~g}^{-1}$.
TABLE 2. Input data at $T_{s}=52^{\circ} \mathrm{C}$

$$
\begin{aligned}
& \text { Specific volume of quenched sample, } \bar{V}_{q-\ldots} \mid 0.4757 \mathrm{~cm}^{3} \mathrm{~g}-1 . \\
& \text { Specific volume of crystallized sample (5 day), } 0.4646 \mathrm{~cm}^{3} \mathrm{~g}-1 \text {. } \\
& \bar{V}_{x} \\
& \text { Volume-temperature derivative of quenched } 1.88 \times 10^{-4} \mathrm{~cm}^{3} \mathrm{gg}^{-1} \mathrm{deg}-1 \text {. } \\
& \text { sample from data above } T_{g}, d \bar{V}_{q}^{+} / d T \text {. } \\
& \text { Volume-temperature derivative of quenched } 1.26 \times 10^{-4} \mathrm{~cm}^{3} \mathrm{~g}^{-1} \mathrm{deg}-1 \text {. } \\
& \text { sample from data below } T_{g}, d \bar{V}_{q}^{-} / d T \text {. } \\
& \text { Volume-temperature derivative of crystal- } 1.18 \times 10^{-4} \mathrm{~cm}^{3} \mathrm{~g}^{-1} \mathrm{deg}^{-1} \text {. } \\
& \text { lized sample from data above } T_{g}, d \bar{V}_{x}^{+} / d T \text {. } \\
& \text { Volume-temperature derivative of crystal- } 1.00 \times 10^{-4} \mathrm{~cm}^{3 \mathrm{~g}}{ }^{-1} \mathrm{deg}-1 \text {. } \\
& \text { lized sample from data below } T_{g}, d \bar{V}_{x}^{-} / d T \text {. }
\end{aligned}
$$

The volume-temperature derivative obtained from eq $(25)$ is

$$
d \bar{V}_{l} / d T=2.199 \times 10^{-4}+5.886 \times 10^{-7} T,
$$

from which is found that $d \bar{V}_{l} / d T=2.505 \times 10^{-4} \mathrm{~cm}^{3} \mathrm{~g}^{-1}$ at $T_{g}=52^{\circ} \mathrm{C}$. Equations extremely close to (25) and (26) would have been obtained had data for temperatures different than $210^{\circ}$ and $240^{\circ} \mathrm{C}$, say $190^{\circ}$ and $260^{\circ} \mathrm{C}$, been employed to construct equations analogous to eq (23) and (24). Values of $\bar{V}_{l}$ computed by using eq (26) are given in table 1 for temperatures between $52^{\circ}$ and $180^{\circ} \mathrm{C}$.

The next step is to calculate the volume-temperature derivative of the pure crystal at $T_{g}$, using eq (17). Inserting the appropriate data from table 2 into this formula, it is found that $d \bar{V}_{c} / d T=$ $0.894 \times 10^{-4} \mathrm{~cm}^{3} \mathrm{~g}^{-1} \mathrm{deg}^{-1}$ at this temperature. Now that $d \bar{V}_{c} / d T$ and $d \bar{V}_{l} / d T$ are determined, the degree of crystallinity of each specimen can be calculated by using eq (8) and (9):

$$
\begin{gathered}
\chi_{q}=(2.505-1.880) \times 10^{-4} /(2.505-0.894) \times \\
10^{-4}=0.388 \\
\chi_{x}=(2.505-1.180) \times 10^{-4} /(2.505-0.894) \times \\
10^{-4}=0.822 .
\end{gathered}
$$

Using these values of $\chi_{q}$ and $\chi_{x}, \bar{V}_{c}$ at $T_{g}$ can be calculated from both eq (8) and (9). Thus,

$$
\chi_{q}=0.388=(0.4856-0.4757) /\left(0.4856-\bar{V}_{c}\right),
$$

and

$$
\chi_{x}=0.822=(0.4856-0.4646) /\left(0.4856-\bar{V}_{c}\right),
$$

from which it is found that $\bar{V}_{c}=0.46007 \mathrm{~cm}^{3} \mathrm{~g}^{-1}$ at $T_{g}$ in each case. A value for $d \bar{V}_{g} / d T$, the volumetemperature derivative of the pure glass, can be obtained at $T_{g}$ by the use of eq (15) and (16), together with the results already obtained. A summary of the results of the calculations at $T_{g}$ is given in table 3. 
TABLE 3. Results of calculations at $T_{g}=52^{\circ} \mathrm{C}$

\begin{tabular}{|c|c|}
\hline $\begin{array}{l}\text { Degree of crystallinity of quenched sample, } \\
\chi_{q} \text {. }\end{array}$ & 0.388. \\
\hline $\begin{array}{l}\text { Degree of erystallinity of crystallized sample } \\
\left(5 \text { day), } \chi_{x} \text {. }\right.\end{array}$ & 0.822 \\
\hline $\begin{array}{l}\text { Volume-temperature } \\
\text { crystalline phase, } d \bar{V}_{c} / d T \text {. }\end{array}$ & $0.894 \times 10^{-1} \mathrm{~cm}^{3} \mathrm{~g}^{-1} \mathrm{deg}^{-1}$. \\
\hline $\begin{array}{l}\text { Volume-temperature derivative of pure } \\
\text { glassy state, } d \bar{V}_{g} / d T \text {. }\end{array}$ & $1.492 \times 10^{-1} \mathrm{~cm}^{3} \mathrm{~g}^{-1} \mathrm{deg}^{-1}$. \\
\hline $\begin{array}{l}\text { Volume-temperature derivative of pure } \\
\text { supercooled liquid, } d \bar{V}_{l} / d T \text {. }\end{array}$ & $2.505 \times 10^{-4} \mathrm{~cm}^{3} \mathrm{~g}^{-1} \mathrm{deg}^{-1}$ \\
\hline $\begin{array}{l}\text { Specific volume of pure supercooled liquid } \\
\text { and glassy state, } \bar{V}_{l}=\bar{V}_{g} \text {. }\end{array}$ & $0.48560 \mathrm{~cm}^{3} \mathrm{~g}-1$ \\
\hline Specific volume of pure crystalline phase, $\bar{V}_{\mathrm{c}-}$ & $0.46007 \mathrm{~cm}^{3} \mathrm{~g}^{-1}$ \\
\hline
\end{tabular}

\subsection{Degree of Crystallinity as a Function of Tem- perature}

The procedure outlined in section 4.3 is followed here. From the previous section, we already have the value $\bar{V}_{c}=0.46007 \mathrm{~cm}^{3} \mathrm{~g}^{-1}$ at $52^{\circ} \mathrm{C}$. At $115^{\circ} \mathrm{C}$, we use eq (19) with the value $\chi_{x}=0.822$ and $\bar{V}_{l}=$ 0.50255 , the latter value calculated from eq (25). It is found that $\bar{V}_{c}=0.46608 \mathrm{~cm}^{3} \mathrm{~g}^{-1}$ at $115^{\circ} \mathrm{C}$. At $-40^{\circ} \mathrm{C}$, using $\chi_{x}=0.822$ and $\chi_{q}=0.388$, together with the values of $\bar{V}_{x}$ and $\bar{V}_{q}$ listed in table 1 , it is found by simultaneously solving eq (20) and (21) that $\bar{V}_{c}=0.45254 \mathrm{~cm}^{3} \mathrm{~g}^{-1}$ and $\bar{V}_{g}=0.47445 \mathrm{~cm}^{3}{ }^{-1}$. Thus, we have three simultaneous equations of the form of eq (18) for the pure crystal covering the interval $-40^{\circ}$ to $+115^{\circ} \mathrm{C}$, the latter being the highest temperature where it is certain $d \chi / d T=0$ :

$$
\begin{aligned}
& \mathrm{A}^{\prime}-40 B^{\prime}+1600 C^{\prime}=0.45254 \\
& A^{\prime}+52 B^{\prime}+2704 C^{\prime}=0.46007 \\
& A^{\prime}+115 B^{\prime}+13225 C^{\prime}=0.46608 .
\end{aligned}
$$

It is found from eq (31 to 33) that

$\bar{V}_{c}=0.45563+0.8079 \times 10^{-4} T+0.874 \times 10^{-7} T^{2}$.

This result may be checked for consistency by calculating $d \bar{V}_{c} / d T$ at $52^{\circ} \mathrm{C}$. The value is $0.899 \times 10^{-4}$ $\mathrm{cm}^{3} \mathrm{~g}^{-1} \mathrm{deg}^{-1}$, which is in good agreement with the value $0.894 \times 10^{-4} \mathrm{~cm}^{3} \mathrm{~g}^{-1} \mathrm{deg}^{-1}$ obtained from eq (17).

The degree of crystallinity of the 5-day crystallized sample is obtained as a function of temperature by inserting values of $\bar{V}_{l}$ and $\bar{V}_{c}$ calculated with eq (25) and (34), together with the values of $\bar{V}_{x}$ listed in table 1, into expression (2) or (20). These operations are conveniently carried out by using the numerical values for $\bar{V}_{l}$ and $\bar{V}_{c}$ listed in table 1 . Values of the degree of crystallinity calculated for the 5 -day crystallized sample from $52^{\circ}$ to $216^{\circ} \mathrm{C}$ are given in table 4 and plotted in figure 5.

The degree of crystallinity of the quenched speciment has been calculated from $52^{\circ}$ to $115^{\circ} \mathrm{C}$ in an analogous way, and the results are indicated in table 4 and figure 5.

\begin{tabular}{|c|c|c|}
\hline Temperature & $\begin{array}{c}\text { Crystallized } \\
\text { sample } \\
\text { (5-day) } \\
\chi_{x}\end{array}$ & $\begin{array}{c}\text { Quenched } \\
\text { sample, } \\
\chi_{x}\end{array}$ \\
\hline $\begin{array}{l}{ }^{\circ} C \\
52 \\
65 \\
80 \\
80 \\
100 \\
115\end{array}$ & $\begin{array}{l}0.822 \\
.822 \\
.822 \\
.822 \\
.822\end{array}$ & $\begin{array}{l}0.388 \\
.386 \\
.386 \\
.387 \\
.388\end{array}$ \\
\hline $\begin{array}{l}130 \\
150 \\
180 \\
190 \\
200\end{array}$ & $\begin{array}{l}.820 \\
.809 \\
.764 \\
.730 \\
.657\end{array}$ & - \\
\hline $\begin{array}{l}210 \\
215 \\
216\left(T^{\prime}{ }_{m}\right)\end{array}$ & $\begin{array}{l}.532 \\
.127 \\
.000\end{array}$ & - \\
\hline
\end{tabular}

TABLE 4. Degree of crystallinity of poly-(chlorotrifluoroethylene) as a function of temperature

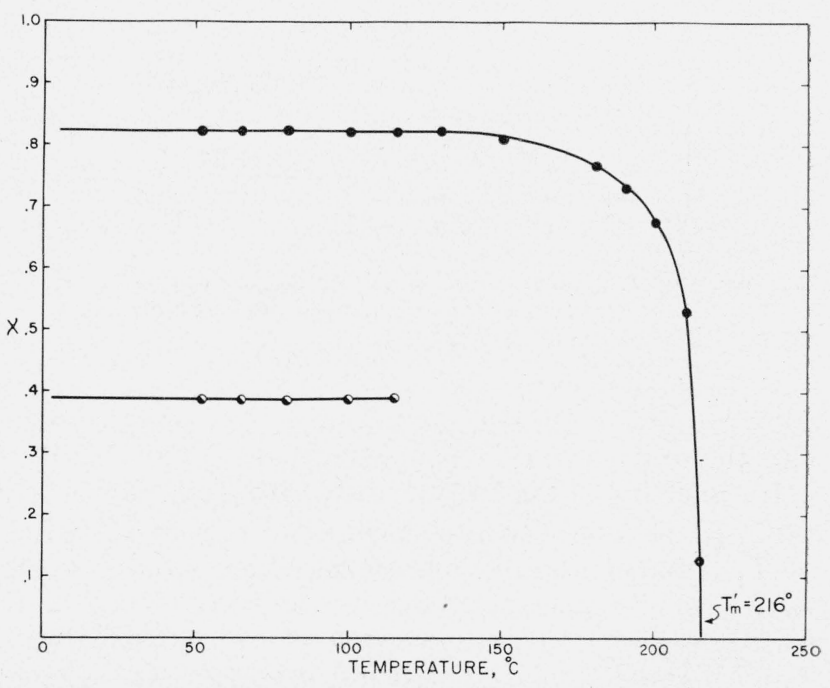

Figure 5. Degree of crystallinity of poly(chlorotrifluoroethylene) as a function of temperature.

- 5-day crystallized specimen; $\mathbf{O}$, quenched specimen.

Values of $\bar{V}_{g}$ may be obtained at various temperatures by repeating the operation used to obtained the value at $-40^{\circ} \mathrm{C}$. The data are accurately represented by the equation

$$
\bar{V}_{g}=0.47884+1.186 \times 10^{-4} T+2.20 \times 10^{-7} T^{2} .
$$

Values of $\bar{V}_{g}$ obtained at various temperatures are given in table 1.

\subsection{Errors}

A rigorous analysis of the errors is prohibitively difficult, but under certain assumptions, a reasonable estimate of the standard deviation associated with the essential results can be made. As indicated earlier, the standard deviation of each of the four measured volume-temperature coefficients at $T_{g}$ is close to 3 percent. Using an equation involving 
first derivatives of the form $\chi_{s}=\left[\left(d \bar{V}_{l} / d T\right)-\left(d \bar{V}_{s}+1\right.\right.$ $d T)] /\left[\left(d \bar{V}_{l} / d T\right)-\left(d \bar{V}_{c} / d T\right)\right]$, where the term in $d \bar{V}_{c} / d T$ is determined by using eq (17), and assuming that the standard deviation of $d \bar{V}_{l} / d T$ is also 3 percent, it is found that the standard deviation is 0.05 for both $\chi_{x}$ and $\chi_{q}$. This estimate is provisional on account of the fact that the error assumed for $d \bar{V}_{l} / d T$ at $T_{g}$ is somewhat arbitrary, but it is believed that the standard deviations quoted for $\chi_{x}$ and $\chi_{q}$ are fairly close to correct. The view that the standard deviation in $\chi$ is small is considerably strengthened by the fact that a straightforward calculation based on second derivatives gives $\chi_{x}$ and $\chi_{q}$ values in close accord with those obtained with the first derivative form (see below). Raising the assumed error in $d \bar{V}_{l} / d T$ to 6 percent, which is certainly an upper limit, increases the standard deviation of $\chi_{x}$ and $\chi_{q}$ to only 0.07 .

Taking the error in $\chi_{x}$ and $\chi_{q}$ to be 0.05 , it is found that the standard deviation in $\bar{V}_{l}$ and $\bar{V}_{c}$ at $T_{g}$ is about $0.0015 \mathrm{~cm}^{3} \mathrm{~g}^{-1}$. Approximately the same value of the error holds for $\bar{V}_{g}$ and $\bar{V}_{c}$ from $T_{g}$ down to $-40^{\circ} \mathrm{C}$, and for $\bar{V}_{l}$ and $\bar{V}_{c}$ up to $115^{\circ} \mathrm{C}$. Above $115^{\circ} \mathrm{C}$, the deviation in $\bar{V}_{l}$ begins to fall, eventually becoming about $0.00040 \mathrm{~cm}^{3} \mathrm{~g}^{-1}$ at $190^{\circ} \mathrm{C}$. The standard deviation in $\bar{V}_{c}$ increases above $115^{\circ} \mathrm{C}$, and reaches a value of about $0.0025 \mathrm{~cm}^{3} \mathrm{~g}^{-1}$ at $200^{\circ} \mathrm{C}$. As a consequence, the standard deviation of the degree of crystallinity, as calculated from eq (2), does not exceed a value of approximately 0.05 at any temperature. The deviation in $\bar{V}_{l}$ at $T_{g}$ is seen to be much less than the value $0.0070 \mathrm{~cm}^{3} \mathrm{~g}^{-1}$, which would have resulted had the usual unguided extrapolation of the liquidus from high temperatures been employed.

It might be thought that the present treatment could be materially improved by including terms of the order of $T^{3}$ and higher in eq (10). There is no basic objection to the use of such higher terms. However, the present analysis, using three constants for describing the specific volume of the liquid and supercooled liquid states, is actually good enough to give roughly correct results even with second derivatives. By taking the second derivative of eq (3) with respect to temperature, we obtain an equation for the degree of crystallinity of the form $\chi_{s}=\left[\left(d^{2} \bar{V}_{l} / d T^{2}\right)-\left(d^{2} \bar{V}_{s}{ }^{+} / d T^{2}\right)\right] /\left[\left(d^{2} \bar{V}_{l} / d T^{2}\right)-\left(d^{2} \bar{V}_{c} /\right.\right.$ $\left.\left.d T^{2}\right)\right]$. Then, using the appropriate formulas in the text to obtain numerical values of $d^{2} \bar{V}_{l} / d T^{2}$ and $d^{2} \bar{V}_{c} / d T^{2}$, and taking the slopes of the volumetemperature coefficients above $T_{g}$ in figure 4 , it is found that the above expression gives $\chi_{x}=0.82$ and $\chi_{q}=0.49$. This is a harsh test of the data, and the good agreement of these values with the ones obtained by using the first derivatives lends credence to the accuracy of the latter. This also shows that terms of $T^{3}$ and higher order in the liquidus equation are not necessary to carry out the analysis to an acceptable degree of accuracy. Extremely precise data in the true liquid range might warrant the use of a $T^{3}$ term in eq (10). All that would be required to evaluate the constant associated with this additional term would be to include another equation of the type of (13) and (14) in the analysis.

\section{Discussion}

\subsection{Degree of Crystallinity and Specific Volume: Comparison With Earlier Results}

The degree of crystallinity calculated for the quenched sample, $\chi_{q}=0.39 \pm 0.05$, which holds for temperatures below $115^{\circ} \overline{\mathrm{C}}$, compares favorably with the value $\chi_{q}=0.35$ obtained by the specificheat method [4]. Owing to the presence of certain approximations inherent in the method of analysis used in the determination based on specific heats, and the approximate nature of that data, it is believed that more reliance may be put in the present result. It is also of interest to note that the specific volume values obtained for the quenched specimen are in good agreement with those published by earlier investigators [8], the difference generally being only about $0.0010 \mathrm{~cm}^{3} \mathrm{~g}^{-1}$.

There is good reason for the general agreement found by various investigators concerning the specific volume and degree of crystallinity of quenched samples $\sim 1.5$ to $3.0 \mathrm{~mm}$ thick, the explanation being found in the details of the kinetics of cristallization [3]. As noted in section 2.3, even 3mm-thick samples removed from a hot mold and "quenched" in air have a specific volume, and hence a degree of crystallinity, not too far removed from that characteristic of an efficiently quenched specimen of similar dimensions.

Based on the results mentioned above, it would appear that the degree of crystallinity of quenched samples $1.5 \mathrm{~mm}$ thick prepared elsewhere, using the technique mentioned in section 2.3 , should be close to the same as was found in the present investigation. However, samples differing significantly in molecular weight, or containing a greatly different number of heterogeneous nuclei, may lead to different results. Agreement with the present work should only be expected in the cases where the method employed to measure the degree of crystallinity is capable of sensing the presence of all the crystalline regions, large and small, and measures the mass fraction of the polymer that is crystalline. The degree of crystallinity of a quenched specimen will increase markedly if it is heated much above $115^{\circ} \mathrm{C}$ for any length of time, so that the degree of crystallinity becomes a function of both time and temperature between $\sim 115^{\circ}$ and $216^{\circ} \mathrm{C}$. It is therefore recommended that comparisons between the results of various types of measurement of the degree of crystallinity not be made in this temperature interval by using quenched samples.

The degree of crystallinity of the 5-day specimen, $\chi_{x}=0.82 \pm 0.05$, cannot be compared directly with the results of earlier work. This follows from the fact that this sample was crystallized considerably longer, and is certainly more highly crystalline, than any used previously. Owing to the reproducibility 
and stability of the 5-day specimen, it is recommended that this type of crystalline sample, rather than one crystallized at a specified cooling rate $[4,5]$, be used in future investigations aimed at a comparison of methods.

A comparison of results that should be free of errors due to sample variation may be made in the case of authors who have determined the degree of crystallinity of two specimens of known specific volume, and therefore are able to compute the specific volume of the pure amorphous and the pure crystalline material. Using degree of crystallinity data on several specimens obtained by the infrared methed, Matsuo [5] has calculated that the specific volume of purely crystalline poly(chlorotrifluoroethylene) at $25^{\circ} \mathrm{C}$ would be $0.4575 \pm 0.0015 \mathrm{~cm}^{3} \mathrm{~g}^{-1}$. This is in excellent agreement with the value $\bar{V}_{c}=0.4577$ $\pm 0.0015 \mathrm{~cm}^{3} \mathrm{~g}^{-1}$ obtained in the present study. ${ }^{8}$ However, for the pure glassy state at the same temperature, we find $\bar{V}_{g}=0.4820 \pm 0.0015 \mathrm{~cm}^{3} \mathrm{~g}^{-1}$, whereas Matsuo gets the much higher value 0.4901 $\pm 0.0006 \mathrm{~cm}^{3} \mathrm{~g}^{-1}$ for the pure "amorphous" component. The latter value cannot be reconciled with the present data.

Support for the validity of the suggested $\bar{V}_{g}$ value may be found in Matsuo's specific-volume data on thin films when considered together with information concerning the kinetics of crystallization. By subjecting a film $0.03 \mathrm{~mm}$ thick to strong quenching, Matsuo [5] was able to produce polymer that had a specific volume of $0.4820 \mathrm{~cm}^{3} \mathrm{~g}^{-1}$ at $25^{\circ} \mathrm{C}$. Now this figure is just that predicted by the present treatment for the specific volume of the pure glassy state at $25^{\circ} \mathrm{C}$, whereas Matsuo's infrared measurements indicated that material of this type was about 25 percent crystalline. Therefore, evidence from another source which indicated that a film treated in this way is essentially noncrystalline, would support the present results. Assuming that such a thin film is quenched through the relatively small temperature interval where the crystallization is quite rapid in a time interval on the order of a tenth of a second or less it is clear from kinetic data [3] that the specific volume of the polymer would never be able to get appreciably below $\bar{V}_{l}$, i. e., it would suffer very little crystallization. Even if one supposed that the degree of crystallinity scale as measured from $\bar{V}_{l}$ was in error by a factor of 2 , the degree of crystallinity of the $0.03-\mathrm{mm}$ quenchedfilm studies by Matsuo could hardly exceed 5 percent, and it is probably considerably less. It is believed that the above argument indicates that the specificvolume value predicted here for the pure glassy state at $25^{\circ} \mathrm{C}$ is more nearly correct than the higher figure given by Matsuo.

\footnotetext{
${ }^{8}$ The unit cell determined from X-ray studies (see [21 and 22]) yields $\bar{V}_{c}=0.47$
$\mathrm{~cm}^{3 \mathrm{~g}-1}$ at $25^{\circ} \mathrm{C}$. However, the estimated error in this figure is in the vicinity of $\mathrm{cm}^{3} \mathrm{~g}-1$ at $25^{\circ} \mathrm{C}$. However, the estimated error in this figure is in the
several percent (private communication from $\mathrm{H}$. S. Kaufmann).
}

\subsection{Practical Method of Determining $\chi$ Near Room Temperature}

It is worth pointing out that the degree of crystallinity of poly (chlorotrifluoroethylene) may readily be measured from a single specific-volume measurement taken near room temperature. For example, using the data in table 1 , it is found that at $20^{\circ} \mathrm{C}$ that

$$
\chi_{s}=\left(0.4814-\bar{V}_{s}\right) / 0.0241 .
$$

Measurements of the specific volume are easily made to $0.0001 \mathrm{~cm}^{3} \mathrm{~g}^{-1}$ in this temperature region. Equations applicable at other temperatures may be readily found by using the data in table 1 .

\subsection{Properties of the Pure Supercooled Liquid, Glassy, and Crystalline Phases}

In the course of calculating the degree of crystallinity of the quenched and crystallized samples, we have indicated passim the manner in which the specific volume of the purely amorphous and purely crystalline phases of this polymer were obtained at various temperatures. The results are summarized in table 1 (figures in ordinary type) and plotted as dashed lines in figure 1 .

The line describing the specific volume of the liquid and supercooled liquid phases as a function of temperature has a definite positive curvature. This is suggested even by the statistical analysis of the points actually observed for the liquid and supercooled region between $260^{\circ}$ and $190^{\circ} \mathrm{C}$, a straight line of the form $\overline{V_{l}}=\alpha+\beta T$ not fitting the data nearly as well as the curved line $\bar{V}_{l}=\alpha+\beta T+\gamma T^{2}$, and thereby being excluded as a proper fit. The more detailed analysis given in the paper, which essentially fixes $\bar{V}_{l}$ at $T_{g}$, using volume-temperature derivatives measured near $T_{g}$, conclusively demonstrates that fitting the liquid and supercooled liquid data with a straight line would have been considerably in error.

The predicted specific volumes of the pure glassy state of this polymer are of interest, especially when compared with those of the pure crystal. Perhaps the most notable point here is that the fractional free volume in the glassy state decreases with lowering temperature. At $T_{g}$, the fraction of free volume in the glass, which is calculated as $\left[\bar{V}_{g}-\bar{V}_{c}\right] / \bar{V}_{g}$, is 0.053 . This quantity falls to 0.046 at $-40^{\circ} \mathrm{C}$. There is little doubt concerning this result, at least from a qualitative standpoint. The difference between the specific volumes of the quenched and crystallized samples becomes considerably less in the same temperature interval to an extent that greatly exceeds the experimental error (note diminishing value of $\bar{V}_{q}-\bar{V}_{x}$ below $T_{g}$ in figure 3 ). This, taken together with the fact that $\chi_{x}$ and $\chi_{q}$, whatever their true values, must certainly be constant below $T_{g}$, then leads to the result that the glass must shrink faster than the crystal. 
The free volume of the glassy state at $T_{g}$ defined in the usual way as $\phi_{g}=\left[\bar{V}_{g}\left(T=T_{g}\right)-\bar{V}_{g}\left(T=0^{\circ} \mathrm{K}\right)\right]$ is found to be $0.023 \mathrm{~cm}^{3} \mathrm{~g}^{-1}$, using eq (35). Actually, this value is a little too high owing to the fact that the volume-temperature derivative of the glass tends toward zero at $0^{\circ} \mathrm{K}$ as depicted in figure 6 , and it is estimated that the true value of $\phi_{g}$ is nearer 0.022 $\mathrm{cm}^{3} \mathrm{~g}^{-1}$. This compares favorably with the value 0.019 to $0.025 \mathrm{~cm}^{3} \mathrm{~g}^{-1}$ found to be consistent with the viscosity data for many linear polymers.

The relatively large additional rise of specific volume in the pure amorphous phase, which begins near $T_{g}$ (compare $\bar{V}_{l}$ and $\bar{V}_{g}$ curves in figure 1), marks the inception of the supercooled liquid state. Thus, the fractional free volume of the glassy state increases slowly and monotonically up to $T_{g}$, where a large excess fractional free volume begins to make its appearance. Factors involved in the relatively small but nevertheless real change of fractional free volume with temperature in the glassy state will be mentioned subsequently.

The relationship between the volume-temperature derivatives of the pure liquid, supercooled liquid, glassy, and crystalline phases over a wide range of temperature is depicted in figure 6 , where the quantities mentioned are plotted on an absolute temperature scale.

The dashed line on the left-hand side of the lower curve in figure 6 represents approximately the type of behavior one would expect at low temperatures for the volume-temperature derivative of the pure crystalline phase, $d \bar{V}_{c} / d T$. This line was sketched in using as a guide a common extension of Grüneisen's theory relating specific heat and the volumetemperature derivative $[15,16,17]$, which states that

$$
d \bar{V}_{c} / d T=\bar{V}^{0} \quad\left[Q_{0} C_{v} /\left(Q_{0}-K E\right)^{2}\right] .
$$

Here $C_{v}$ is the specific heat at constant volume, $E$ the energy of the intermolecular vibrations in the system, $\bar{V}$ the volume at $0^{\circ} K, Q_{0}$ a constant usually expressed in calories per mole, and $K$ a constant that depends on the details of the intermolecular potential. ${ }^{9}$ At low temperatures $Q_{0}$ is considerably in excess of $K E$, causing the temperature dependence of $d \bar{V}_{c} / d T$ to vary directly as $C_{v}$. Now according to the theories of Einstein [18] and Debye [19], the specific heat of a crystal is zero at $0^{\circ} \mathrm{K}$, rises at first in an increasingly steep manner, and then begins to level off at its "classical" value near a characteristic temperature, $\theta$. Hence, from eq (37) we must expect the volumetemperature derivative of the crystalline phase of the polymer to behave in a qualitatively similar way. It has been assumed in sketching in the dashed line in figure 6 that $\theta$ is $200^{\circ} \mathrm{K}$, a reasonable value for a molecular crystal. In contrast to the models with 3 degrees of freedom treated by Einstein and Debye, it is likely in the present case that only the two The constant $Q_{0}$ is known to be related to the melting temperature [16], and
it is therefore presumably related to the lattice energy. $Q_{0}$ is generally of the order of magnitude of $10^{5}$ calories/mole for metals, and $K$ of the order two.

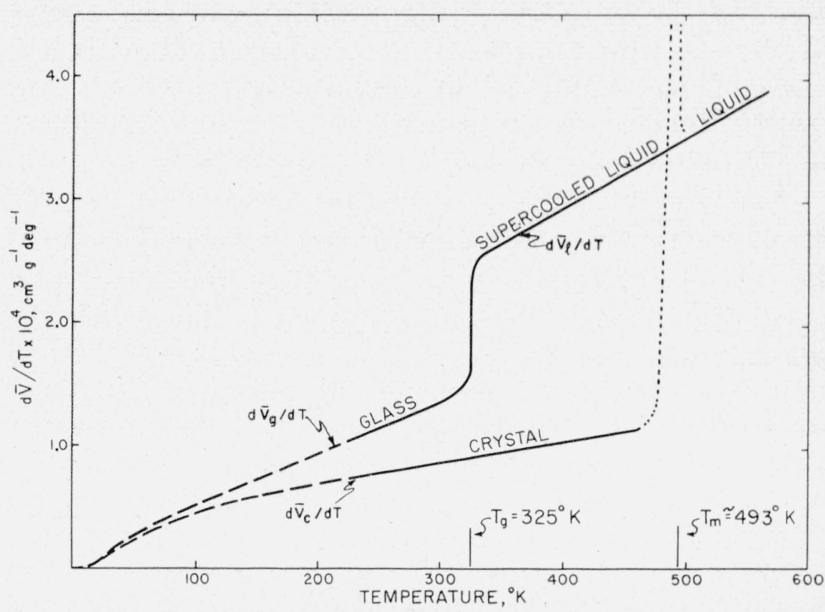

FIgurE 6. Estimated volume-temperature derivatives of the pure supercooled liquid, glassy, and crystalline states of poly (chlorotrifluoroethylene) as a function of temperature (Kelvin scale)

melting behavior to be expected for the hypothetical completely crystalline phase.

degrees of freedom transverse to the long molecular axis are connected with the expansion of the polymer crystal, and that more than one $\theta$ temperature is involved, but this does not alter the qualitative description given. The gradual increase of $d \bar{V}_{c} / d T$ evident in the classical region may be partly due to a continued rise of $C_{v}$, but it seems likely that the diminishing value of the term $\left(Q_{0}-K E\right)^{2}$ arising from the increase of $E$ with temperature is also important. To the extent that the latter term is responsible for the moderate increase of $d \bar{V}_{c} / d T$ in this region, the expansion of the polymer crystal is attributable to the tendency of thermal agitation (as measured by $E$ ) to overcome lattice forces (as represented in at least an approximate way by $\left.Q_{0}\right) .{ }^{10}$

The volume-temperature derivative, $d \bar{V}_{g} / d T$, of the pure glassy state in the region below $233^{\circ} \mathrm{K}$ has been sketched in figure 6 with essentially the same concepts in mind. The value $\theta=175^{\circ} \mathrm{K}$ was used. Thus the glassy state has been regarded as having an "average" intermolecular potential, so that it is theoretically permissible to assign $\theta, Q_{0}$, and $K$ values to it. The construction of the dashed line representing $d \bar{V}_{g} / d T$ at low temperatures in figure 6 is in accord with the experimental observation [8] that the length-temperature coefficient of quenched poly(chlorotrifluoroethylene) shows a continuous rise at low temperatures, which tends to level off at around $213^{\circ} \mathrm{K}$. This suggests a $\theta$ temperature of around $150^{\circ}$ to $200^{\circ} \mathrm{K}$.

Considering the points mentioned above concerning the low-temperature behavior, together with the results obtained in a straightforward way from the experimental data at higher temperatures (solid lines in figure 6 ), there is no doubt that starting at very low temperatures, the volume-temperature deriva-

10 The authors thank R. K. Kirby for many helpful discussions concerning the volume-temperature behavior of solid bodies. 
tives of the pure crystalline and pure glassy states diverge as the temperature increases. The divergence of the volume-temperature derivatives is, of course, related to the previously mentioned increase of fractional free volume in the glassy state.

The question must now be raised as to why $d \bar{V}_{g} / d T$ exceeds $d \bar{V}_{c} / d T$. Certainly a part of this effect must be due to differences in $Q_{0}$ and $\bar{V}^{0}$ for the glassy and crystalline states. $\bar{V}^{0}$ is certain to be larger for the glassy state than the crystalline one owing to the less dense nature of the former; the polymer molecules are disordered in the glassy state and therefore occupy more space than they do in the crystal. This factor alone will cause $d \bar{V}_{q} / d T$ to exceed $d \bar{V}_{c} / d T$ by several percent. Also, it is likely that $Q_{0}$ is smaller for the more open structure of the glassy state, again leading to a volume-temperature derivative for the glassy state exceeding that of the crystalline. Differences in $C_{v}$, arising at least in part from the existence of different $\theta$ temperatures for the two states, must also contribute. It is worth pointing out in this connection that the precise specific-heat data of Bekkedahl and Matheson [20] on natural rubber in the completely amorphous (glassy) and partially crystalline conditions reveal that $C_{p}$ for the glassy phase everywhere (except at $0^{\circ} \mathrm{K}$ ) exceeds that of the crystalline. These data imply that $C_{v}$ (glass) $>$ $C_{v}$ (crystal). Their plot of $C_{p}$ versus $T$ bears a remarkable resemblance to the volume-temperature derivative plots shown in figures 2 and $6 .{ }^{11}$

In view of the foregoing, it is not surprising that approximation (7) fails when subjected to a rigorous test, because there are excellent theoretical reasons for believing that $d \bar{V}_{g} / d T$ must exceed $d \bar{V}_{c} / d T$, except at $0^{\circ} \mathrm{K}$, where they are both zero. It is clear from the present study that this approximation should be avoided whenever attempting a precision calculation of the degree of crystallinity, or in estimating the properties of pure phases.

Evidence based on dielectric data shows that the crystalline phase is orientationally disordered to some extent down to low temperatures, and that this disorder increases as the temperature is raised [2]. However, there is no indication from either the dielectric or specific-volume measurements that the gradually increasing degree of disorder culminates in a first-order phase transition in the crystalline state anywhere in the range $-40^{\circ}$ to $+216^{\circ} \mathrm{C}$. The $\bar{V}_{c}$ data thus refer to a crystal that possesses a certain degree of imperfection due to molecuiar motion.

\subsection{Melting Process}

The semicrystalline 5-day sample exhibits melting over a wide range of temperature. This is evident in figures 1,2 , and 5 . The broad nature of the melting

\footnotetext{
${ }^{11}$ A plot of $C_{p}$ versus $T$ should quite generally be expected to strongly resemble a plot of $d \bar{V} / d T$ versus $T$ for corresponding states. The rough specific-heat data for crystallized poly(chlorotrifiuoroethylene) [4] bear a marked similarity to the $d \bar{V}_{x} / d T$ data shown in figure 2. Unfortunately, the comparison cannot be carried further because the specific-heat data are faulty below $T_{g}$ for the quenched specimen, the glass transition having been overlooked as the result of a calorimeter start-up effect.
}

phenomenon in this polymer may be attributed to the presence of small crystallites that melt well below the equilibrium melting temperature. The smallest of these begin to melt at about $120^{\circ} \mathrm{C}$, whereas the largest and most perfect crystallites in the sample, which are sufficient in number to appreciably affect the volume, melt at the quasi-equilibrium melting temperature, $T_{m}^{\prime}=216^{\circ} \mathrm{C}$. (Somewhat larger crystallites of insufficient number to be detected may persist above $T_{m}^{\prime}$ ) As indicated earlier, the latter figure is still below the equilibrium melting temperature, $T_{m}$. Even then, it is worth noting that the 5-day specimen melts above the value $210^{\circ}$ to $212^{\circ} \mathrm{C}$ frequently quoted in the literature [1, 4, 21, 22]. The last half of the sample melts in a temperature interval of about $6{ }^{\circ} \mathrm{C}$. The fractional free volume in the liquid at $T_{m} \cong 220^{\circ} \mathrm{C}$, as calculated by using $\left(\bar{V}_{l}-\bar{V}_{c}\right) / \bar{V}_{l}$, is 0.11 . This is to be compared with the value 0.053 at $T_{g}=52^{\circ} \mathrm{C}$.

\subsection{Glass Transition}

The glass transition in a semicrystalline polymer has been treated in this paper as if it occurred abruptly at the intersection of two curved lines, one representing the volume-temperature relation of the system glass plus crystal below $T_{g}$, and the other the system supercooled liquid plus crystal above $T_{g}$. In any such analysis it is necessary to avoid the use of data too close to the nominal glass temperature owing to the fact that the glass transition, which takes place in the amorphous part of the polymer, is not actually abrupt. Proof of the finite breath of the transition is not lacking in the present case. For instance, the specific volume of the quenched sample undergoes a marked premonitory increase beginning $10^{\circ}$ to $15^{\circ} \mathrm{C}$ below $T_{g}$. The basically somewhat diffuse character of this transition in completely amorphous bodies was clearly pointed out by Kauzmann in his review paper [23] on the nature of the glassy state. However, there is no basic thermodynamic objection to treating this transition in both completely amorphous and semicrystalline media as if it took place abruptly at a certain temperature corresponding to the intersection of two curved lines.

The method used to obtain $T_{g}$, which consisted of plotting $\left(\bar{V}_{q}-\bar{V}_{x}\right)$ against $T$ and noting the point of intersection, requires comment. This procedure has the advantage of not requiring an explicit knowledge of the form of the two curved lines that make the two intersecting volume-temperature curves of each sample. However, this method would have to be applied with caution if the glass temperature varied with the degree of crystallinity. It is possible to show for poly(chlorotrifluoroethylene) that $T_{g}$ does not depend markedly on the degree of crystallinity. By fitting the data given in table 1 describing the specific volume above and below $T_{g}$ for a given semicrystalline specimen to an equation of the form $\bar{V}_{s}=a+b T+c T^{2}$, and simultaneously solving the two equations, it was shown that the glass temperatures of the two samples were within $1.2^{\circ} \mathrm{C}$ of each other. This shows that the glass-transition temperatures of 
the two semicrystalline specimens are identical within experimental error, and justifies the use of the plot of $\left(\bar{V}_{q}-\bar{V}_{\dot{x}}\right)$ versus $T$ that was employed to obtain the reported value $T_{0}=52^{\circ} \mathrm{C}$. Data between $-40^{\circ}$ and $+25^{\circ} \mathrm{C}$, and $+65^{\circ}$ and $+115^{\circ} \mathrm{C}$ were used in this analysis, and all curve-fitting was accomplished by using least-squares.

Precisely the same input data lead to $T_{q}$ values of $50^{\circ}$ and $45^{\circ} \mathrm{C}$ for the samples if an equation of the form $\bar{V}_{s}=a+b T$ is used with a least-squares fit to represent the specific-volume-temperature relations above and below $T_{g}$. The value $T_{g}=52^{\circ} \mathrm{C}$ is to be preferred owing to the fact that it is based on an analysis that admits of the curvature inherent in the experimental specific-volume-temperature data. The apparent difference found for the glass-transition temperature of the two specimens using the "straightline" analysis does not mean that $T_{n}$ actually varies with $\chi$ for this homopolymer, but is simply an artificial result arising from the inability of a straight line to accurately represent the data even at temperatures well removed from the glass transition.

Washington, November 1, 1957.

\section{References}

[1] F. P. Price, J. Am. Chem. Soc. 74, 311 (1952).

[2] J. D. Hoffman, A. H. Scott, A. J. Curtis, D. J. Scheiber, and J. I. Lauritzen, Jr., (manuscript in preparation).

[3] J. D. Hoffman and J. J. Weeks (manuscript in preparation).

[4] J. D. Hoffman, J. Am. Chem. Soc. 74, 1696 (1952).

[5] H. Matsuo, J. Polymer Sci. 25, 234 (1957).

6] H. Matsuo, Bul. Chem. Soc. Japan 30, 593 (1957)

[7] F. P. Price, J. Chem. Phys. 19, 973 (1951).

[8] L. Mandelkern, G. M. Martin, and F. A. Quinn, Jr., J. Research NBS 58, 137 (1957) RP2703.

[9] R. B. Sosman, Properties of silica, p. 307 (Chemical Catalogue Co., New York, N. Y., 1927).

[10] W. Souder and P. Hidnert, BS Sci. Pap. 21, 1 (19261927) S524.

[11] L. Mandelkern, Chem. Revs. 56, 903 (1956).

[12] J. D. Hoffman and J. J. Weeks (manuscript in preparation).

[13] R. F. Boyer, J. Appl. Phy. 25, 185 (1954).

[14] R. F. Boyer, Changement de Phases, Compt. rend. 2d reunion de chemie physics, p. 384 (1952).

[15] B. Dayal, Proc. Indian Acad. Sci. 20 (1944)

[16] Am. Inst. Physies Handbook, p. 4-63 (McGraw-Hill Bock Co., New York, N. Y., 1957).

[17] W. Hume-Rothery, Proc. Phys. Soc. (London) 5z, 209 (1945).

[18] A. Einstein, Ann. physik. 22, 180 (1907); 34, 170 (1911).

[19] P. Debye, Ann. physik. 39, 789 (1912).

[20] N. Bekkedahl and H. Matheson, J. Research NBS 15, 503 (1935) RP844.

[21] H. S. Kaufman, Tech. Assoc. Pulp Paper Ind. 37, 140 (1954)

[22] H. S. Kaufman, J. Am. Chem. Soc, 75, 1447 (1953)

[23] W. Kauzmann, Chem. Revs. 43, 219 (1948). 